\title{
Analysis of Polarization Independent Diffused QW Optical Amplifiers
}

\author{
Wallace C.H. Choy \\ Department of Electrical and Electronic Engineering, University of Hong Kong, Hong Kong \\ (852) 2857-8485, (852) 2559-8738, wchchoy@hkucc.hku.hk
}

\begin{abstract}
The features of polarization independence of optical amplifiers achieved by using diffused $Q W$ are discussed. Our theoretical results successfully explain why polarization independence can achieve in the long wavelength tail of the modal gain and absorption coefficient but not at photon energies above the transition edge. This explanation applies to other tensile-strained $Q W$ for polarization independent applications. The understanding is crucial for optimizing polarization independent devices.
\end{abstract}

\section{TEXT}

There is a well-known inherent feature of QW structures for devices applications, which is TE and TM polarization dependence, due to the non-degeneracy of heavy hole (HH) and light hole (LH) in the growth direction. In this paper, new simulation results are analyzed and discussed for the diffused QW (DFQW) structure which has been theoretically [1] and experimentally [2] demonstrated for providing polarization independent (PI) optical gain. The new theoretical TE and TM gain spectra before and after QW interimixing are in very good agreement with the experimental results in ref [2]. As shown in Fig. 1, the as-grown QW structure is lattice-matched $\mathrm{In}_{0.53} \mathrm{Ga}_{0.47} \mathrm{As}$ (well)/ $\mathrm{In}_{0.74} \mathrm{Ga}_{0.26} \mathrm{As}_{0.57} \mathrm{P}_{0.43}$ (barrier) structure and the well width considered is $6 \mathrm{~nm}$. In the as-grown $\mathrm{QW}$ structure, the eigenstates of $\mathrm{LH}$ and $\mathrm{HH}$ are non-degenerate at $\mathrm{k}_{/ /}=0$ with an energy difference of $21 \mathrm{meV}$. The TE and TM gain spectra thus clearly separate from each other because the optical matrix element (OME) of TE gain is non-zero for both the LH and HH subbands, while the OME of TM gain is non-zero only for the LH subbands and zero for HH subbands [3]. When QW diffused with a diffusion length of group V material $\left(\mathrm{L}_{\mathrm{d}}^{\mathrm{V}}\right)$ increases to $1.8 \mathrm{~nm}, \mathrm{HH} 1$ and $\mathrm{LH} 1$ subbands move close to each other with less than $3 \mathrm{meV}$ energy difference, leading to polarization independent optical gain. The TE and TM gain of the PI-DFQW merge together at the long wavelength tail $(>1460 \mu \mathrm{m})$. However, they split in the shortwavelength region $(<1460 \mu \mathrm{m})$, which is an important feature in a PI device. Our theoretically result shown that join density of state (JDOS) is the main factor responsible for the difference in TE and TM gain. In PI-QW devices, since the mass of $\mathrm{HH}$ parallel to the plane $\left(\mathrm{m}_{\mathrm{hh} /}\right)$ not equals the mass of $\mathrm{LH}$ parallel to the plane $\left(\mathrm{m}_{\mathrm{h} /} /\right)$, the JDOS at photon energies above the transition edge becomes polarization dependence. By assuming $\mathrm{m}_{\mathrm{ph} /}=$ $\mathrm{m}_{\mathrm{lh} / \mathrm{h}}$, we have calculated that the JDOS of transition between first state in conduction band and first state in $\mathrm{HH}$ $(\mathrm{Cl}-\mathrm{HH} 1)$ and $\mathrm{C} 1-\mathrm{LH} 1$ transition obtained in this way are closer in value for a wide range of value of $\mathrm{k}_{/ /}$. We also calculated the TE and TM gain spectra by assuming $\mathrm{m}_{\mathrm{hh} /}=\mathrm{m}_{\mathrm{l} / / .}$. They are close in value in a wide range of wavelength and difference in TE and TM gain above $1460 \mu \mathrm{m}$ is very small. This shows that by putting the $\mathrm{HH}$ and LH's effective mass in $x-y$ plan the difference in TE and TM gain can be eliminated. To conclude, we successfully explain why the TE and TM gain of polarization independent DFQW can only merge at long wavelength tail of the gain spectrum when enough tensile strain is generated by quantum well intermixing to make $\mathrm{HH} 1$ and $\mathrm{LHI}$ degenerate in energy. The main reason for this phenomenon is that, in $\mathrm{QWs}, \mathrm{m}_{\mathrm{hh} / /} \neq \mathrm{m}_{\mathrm{l} / /}$ in the $x-y$ plane. This problem is difficult to remove, as it is an intrinsic property of $\mathrm{QW}$ structures. This explanation not only applies to tensile strained DFQWs but also to other tensile strained QWs designed for PI application.

\section{REFERENCES}

[1] W.C.H. Choy, H. Feng, S.K. Kam, and E.H. Li, "The effect of composition modification on the optical polarization independence in semiconductor strain quantum wells", Mat. Res. Soc. Symp. Proc. vol. 417, pp.277-282 (1996)

[2] J.J. He, S. Charbonneau, P.J. Poole, G.C. Aers, Y. Feng, E.S. Koteles, R.D. Goldberg, and I.V. Mitchell, "Polarization insensitive InGaAs/InGaAsP/InP amplifiers using quantum well intermixing", Appl. Phys. Lett., vol. 69, pp.562-564 (1996)

[3] W.C.H. Choy, "Tailoring Light- and Heavy- holes of GaAsP/AlGaAs Quantum Wells by Using Interdiffusion for Polarization Independent Amplifier Applications", IEEE J. Quantum Electron., vol. 36, pp. 164-174 (2000)

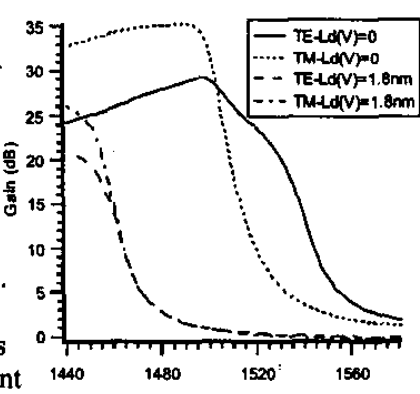

Fig. 1 